\title{
Aplicación de la Teoría Crítica de Raza en la organización y representación del conocimiento
}

Application of Critical Race Theory to knowledge organization and representation

\author{
Daniel Martínez-ÁviLA (1), Marcio Ferreira (1), José L. MAGro (2) \\ (1) Department of Information Science, São Paulo State University - UNESP, Av. Higyno Muzzi Filho, 737, \\ Marília (17525-900) - São Paulo - Brazil, dmartinezavila@marilia.unesp.br, soimers@marilia.unesp.br. \\ (2) The Graduate Center (CUNY), 365 5th Avenue, New York, NY, USA, jmagro@gradcenter.cuny.edu
}

\section{Resumen}

Se introducen las características y posibilidades metodológicas de la Teoría Crítica de Raza aplicada a la organización y representación del conocimiento, y más específicamente a los principales sistemas de clasificación bibliográficos utilizados en Brasil. Para ello, se identifican las clasificaciones bibliográficas como instituciones; y se esbozan los principales puntos de un proyecto de reemplazo de instancias de racismo por alternativas antirracistas.

Palabras clave: Teoría Crítica de Raza. Clasificaciones bibliográficas. Metodología. Organización del conocimiento. Aspectos éticos.

\section{Introducción}

Los sesgos y problemas éticos en las clasificaciones bibliográficas y otros sistemas de organización y representación del conocimiento son un aspecto que ha sido ampliamente expuesto en la literatura de las últimas décadas (por ejemplo, Berman, 1971; Foskett, 1971, 1984; Afolabi, 1992; Olson \& Ward, 1997; Olson, 2000, 2001a, 2002; Olson \& Schlegl, 2001; Milani et al., 2014). El paso de una hegemonía positivista hacia unas posiciones más pragmatistas ha permitido el rechazo cada vez más abierto de valores como la neutralidad y la objetividad en pos de la reivindicación de la explicitación de los puntos de vista en los procesos de organización y desarrollo de sistemas (por ejemplo, García Gutiérrez, 2002; 2007; 2011; Martínez-Ávila \& Guimarães, 2013; García Gutiérrez \& Martínez-Ávila, 2014). En términos más técnicos, Birger Hjørland lo expresa del siguiente modo (Hjørland, 2007 , p. 8 , traducción propia):

\footnotetext{
La teoría dominante en la biblioteconomía y ciencia de la información [LIS] ha sido que los vocabularios normativos representan soluciones 'neutrales', 'objetivas' que simplemente proporcionan sistemas de información más eficientes. Podemos denominar esta visión como 'visión positivista' y contrastarla con una 'visión pragmática' en la que cualquier vocabulario controlado tiende a favorecer al-
}

\begin{abstract}
In this paper we introduce the characteristics and methodological possibilities of applying the Critical Race Theory to knowledge organization and representation, and more specifically to the bibliographic classifications that are used in Brazil. We identify these classifications as institutions and outline the main points of a project of replacement of any instances of racism by anti-racist alternatives.
\end{abstract}

Keywords: Critical Race Theory. Bibliographic classifications. Methodology. Knowledge organization. Ethical aspects.

gunos tipos de consultas a la vez que hace otros tipos de consultas más difíciles de contestar.

Sin embargo, en la práctica, ese tipo de factores y sesgos que favorecen unos tipos de consultas sobre otros (perjudicando también a unos usuarios concretos sobre otros) están lejos de seguir criterios pragmáticos basados en el estudio de las características de las comunidades a las que sirven. Más bien, tal como índica Furner (2007, p. 154, traducción propia), "todo esquema de clasificación puede ser interpretado como sesgado en algún respecto, donde el sesgo es el inevitable reflejo de sus diseñadores", es decir, reflejando los sesgos, intereses y características de la comunidad dominante que los engendra, que en el caso de los principales sistemas de organización del conocimiento predominantes es blanca, étnicamente europea, burguesa, cristiana protestante, heterosexual, sin discapacidades físicas y masculina (1) (Olson, 2001b).

En este caso, los problemas prácticos de los usuarios pertenecientes a otros grupos diferentes de esta clase dominante, como por ejemplo las comunidades afrodescendientes, no se limitan únicamente a inofensivas consultas técnicamente más difíciles de contestar, sino que también pueden abarcar aspectos sociales mucho más sensibles como por ejemplo representaciones deformadas y la creación y continuación de estereotipos, omisión e invisibilización 
de la comunidad o sus características, uso de lenguaje ofensivo para la comunidad, y agrupamiento o asimilación de la comunidad con otros grupos cuyo único nexo en común es compartir el estatus de "otro" en relación a la clase dominante.

Los casos de racismo en sistemas y otras instituciones de organización y representación del conocimiento han sido bien documentados en la citada bibliografía. En este sentido, tal como indican Bowker y Star (1999), las clasificaciones deben abandonar su estatus de herramientas neutrales y escondidas para pasar a ser elementos activos de trabajo ético y político. Así pues, adoptando una postura crítica, ética y emancipadora, los fundamentos y posibilidades de la Teoría Crítica de Raza (TCR) podrían suponer un perfecto cuadro de referencia teórico y metodológico para estudiar y eliminar estas instancias de racismo en sistemas utilizados por comunidades afrodescendientes.

En el presente trabajo se busca profundizar en las principales características y posibilidades metodológicas para la organización y representación del conocimiento de la TCR, y esbozar su posible aplicación en el contexto de dos de los principales sistemas de clasificación utilizados en Brasil: la Clasificación Decimal de Dewey (CDD) y la Clasificación Decimal Universal (CDU).

\section{Uso de la Teoría Crítica de Raza}

La TCR surgió a mitad de los años 1970s en los Estados Unidos, englobando principalmente abogados, activistas e investigadores en el área de Derecho que propusieron nuevas teorías y estrategias para combatir el racismo que había ganado terreno desde el parón en avances en derechos civiles de los años 1960s. (Delgado \& Stefancic, 2001, p. 3-4). En la actualidad, tal como indican Delgado y Stefancic (pp. 2-5, traducción propia), "el movimiento de la Teoría Crítica de Raza es una colección de activistas e investigadores interesados en estudiar y transformar la relación entre raza, racismo y poder", que también tiene relación con otros movimientos como los estudios legales, el feminismo radical, filósofos y teóricos europeos como Antonio Gramsci y Jacques Derrida, la tradición radical americana de Sojourner Truth, Frederick Douglass, W. E. B. Du Bois, Cesar Chavez, Martin Luther King, Jr., y los movimientos Black Power y Chicano de los 1960s y principios de los 1970s.

Derrick Bell, profesor de Derecho en la Universidad de Nueva York, es considerado el padre intelectual del movimiento, mientras que otras figuras importantes incluyen a Alan Freeman, Kimberlé Crenshaw, Angela Harris, Charles Lawrence y Patricia Williams en su vertiente tradicional, Neil Gotanda, Eric Yamamoto y Mari Matsuda como autores asiáticos, Robert Williams como principal autor indio, y Richard Delgado, Kevin Johnson, Margaret Montoya, Juan Perea y Francisco Valdés como principales autores latinos.

En el contexto de la biblioteconomía y ciencias de la información, la aplicación de la TCR ya ha sido discutida en la archivística (Dunbar, 2006), Internet (Brock, 2009), servicios bibliotecarios (Kumasi, 2012), clasificaciones bibliográficas (Furner \& Dunbar, 2004) y la versión en inglés de la CDD (Furner, 2007). Jonathan Furner puede ser considerado el principal precursor de la TCR en la organización y representación del conocimiento.

\subsection{Características de la Teoría Crítica de Raza}

No existen unas características uniformes y únicas en los autores que trabajan con TCR. Harris (1994) y Bell (1995) resumen estas diferencias en dos corrientes: la que se destaca por su compromiso con la crítica radical (normativamente deconstructivista), y la que se destaca por su compromiso con la emancipación radical (normativamente reconstruccionista). Común a toda la TCR es el objetivo de erradicar la injusticia racial, primero revelando su naturaleza institucional, y segundo especificando y conduciendo la acción radical necesaria para reemplazar las instituciones racistas por alternativas antirracistas (Furner, 2007, p. 144). En el caso del trabajo de Furner, una de las instituciones que son identificadas como racistas es la CDD, que en su caso es identificada como una institución de información. Enlazándolo con las teorías de justicia social, Furner ( $p$. 155) también indica que los esquemas de clasificación pueden ser evaluados si son tratados como generadores de distribuciones de bienes conceptuales. Otros ejemplos de instituciones de información que también podrían ser objeto de estudio pueden ser cualquiera de los estándares, reglas y recomendaciones para catalogadores y bibliotecarios. En nuestro caso, hemos optado por continuar con la línea de trabajo de Furner en clasificaciones bibliográficas, identificando como instituciones racistas la aplicación de la CDD y CDU en Brasil, e introduciendo la TCR al discurso brasileño de organización y representación del conocimiento.

En ambos casos, las características comunes de esta teoría son compatibles con las visiones pragmatistas en organización y representación 
del conocimiento en las que se rechaza la existencia y posibilidad de decisiones libres de valores en cualquier proceso o práctica profesional, que incluyen desde la toma de decisiones, elaboración de normas, leyes, etc. hasta el diseño de clasificaciones y otros sistemas. En este sentido, la TCR trabaja con la tesis de la "construcción social", que afirma que las razas son únicamente productos de pensamiento y relaciones sociales. Las razas, en palabras de Delgado y Stefancic (2001, p. 7, traducción propia), "no son objetivas, inherentes o fijas, no corresponden con ninguna realidad biológica o genética; más bien, las razas son categorías que la sociedad inventa, manipula o retira cuando es conveniente".

\subsection{Sobre el concepto de raza y racismo}

El concepto de "raza" no está demostrado como algo natural y absoluto desde un punto de vista biológico. Lo que es normalmente distinguido como "raza" es una agrupación artificial de elementos en función de criterios de semejanza fisiológica (y muy comúnmente también confundidos con atributos étnicos y culturales como lenguaje, religión, etc., e incluso geográficos) comúnmente utilizada con fines identitarios 0 discriminatorios.

No obstante, desde el punto de vista social constructivista, el hecho de que un concepto no sea natural no significa que no sea real. Es reconocido que la posición eliminativista —en la que el concepto de raza es eliminado o ignorado por no ser natural- no parece ser una solución efectiva mientras existan identidades que son construidas tanto activa como pasivamente por terceros con fines discriminatorios. El hecho de que no se pueda demostrar científicamente que existe una línea natural que distingue elementos de una raza de los de otra no ha prevenido que individuos hayan adoptado criterios artificiales para identificarse con un grupo y distinguirse de "otros" (los cuales estarían definidos, siguiendo a Derrida, por sus diferencias).

Un acto puede considerarse racista si trata injustamente a un individuo con motivo de ser percibido o identificado con una raza. En ese sentido, la TCR sí reconoce el derecho del individuo de auto-identificación con una raza, especialmente cuando es utilizado para explicar los actos discriminatorios motivados por los aspectos definitorios del grupo al que fue adscrito.

Dicho esto, la TCR no puede ser calificada ni de racista ni de ser equivalente al racismo que es ejercido contra los que son percibidos como noblancos. David Ingram indica que "afirmar 'orgullo negro' no es equivalente a afirmar 'orgullo blanco', ya que el primero -al contrario que el segundo- es una estrategia defensiva dirigida a rectificar un estereotipo negativo" (Ingram, 2004, p. 55, traducción propia). La TCR busca rectificar unas situaciones de injusto privilegio blanco a través de instituciones en sociedades, como por ejemplo la estadounidense o la brasileña, donde, a pesar de existir un porcentaje amplio de personas afrodescendientes, los sistemas no son diseñados para "favorecer sus tipos de consultas" o reflejar sus puntos de vista.

Tal como afirmaba A. C. Foskett, comentando el citado trabajo de Sanford Berman (1971), "la Lista de Encabezamientos de Materia de la Biblioteca del Congreso (LCSH) representa un punto de vista imperialista que puede ser probado como vergonzoso en una biblioteca africana" (Foskett, 1971, p. 121). Este punto de vista que menciona Foskett quizás podría ser pragmáticamente aceptable en un sistema diseñado exclusivamente para blancos imperialistas (aunque todavía podría plantear objeciones éticas dentro de su propia comunidad), pero no para comunidades con un considerable número de usuarios afros.

La TCR busca describir y explicar esas instancias de racismo y actuar desde un triple compromiso entre sus características: un compromiso epistemológico con la construcción social (de conceptos como la raza), un compromiso ético con la justicia social, y un compromiso metodológico con acciones radicales de tanto tipo físico como intelectual (Furner, 2007, p. 146).

\subsection{Propuestas metodológicas de la Teoría Crítica de Raza para la ORC}

Dentro de una postura epistemológica social constructivista, que podría coincidir con posiciones pragmatistas y postestructuralistas en el análisis y discusión de los datos, la TCR además presenta frecuentemente las siguientes técnicas en la construcción de sus textos: uso de la primera persona, storytelling, narrativa, contra-historias y contra-narrativas, alegorías, tratamiento interdisciplinar de la ley y todo tipo de usos creativos.

Como ejemplo de estas técnicas, Derrick Bell, en uno de sus relatos académicos más celebres, The space traders, presenta el hipotético escenario ético de una visita de extraterrestres a los Estados Unidos los cuales ofrecen cien años de prosperidad para la nación a cambio del sacrificio de toda la población negra, para lo cual el total de la población se somete a un referéndum (Bell, 1992). Explicando su método, Bell indica que prefiere (1995, p. 902, traducción propia): 
Usar las historias como un medio de comunicar puntos de vista a aquellos que mantienen otros puntos de vista muy diferentes, en un tema tan emocionalmente cargado como es la raza. Las personas disfrutan con las historias y por eso muchas veces dejarán de lado sus creencias, escucharán las historias, y entonces compararán sus puntos de vista no con el mío, sino con el de aquellos que son expresados en la historia

Tal como indica Brown (1995, p. 514, traducción propia), siendo suscrito por Dunbar en su aplicación de la TCR al campo de la archivística (2006, p. 113), algunos de los puntos más importantes del uso de las contra-narrativas como metodología son los siguientes:

Los teóricos críticos de raza usan la narrativa en un esfuerzo de auto-consciencia para incluir las voces de la gente de color que tradicionalmente ha sido excluida de la ciencia legal convencional y 'apropiada'.

El uso de la narrativa reta el paradigma tradicional de la meritocracia en la academia al intentar subvertir lo que es visto como pretensiones de 'objetividad', 'neutralidad', 'meritocracia', y 'ceguera al color'.

Puede decirse, por tanto, que el uso de historias y contra-narrativas es un mecanismo que es utilizado para dar voz a grupos marginalizados y oprimidos cuyos discursos han sido tradicionalmente silenciados, especialmente en la academia. Por otra parte, también es una forma de construir realidades sociales alternativas, tan artificiales como las narrativas reales oficiales, y que sirven como llamada de atención sobre la injusticia social de éstas. Tal como resume Dunbar (2006, p. 115, traducción propia), Delgado (1989, pp. 2414-2416) también indica diversas razones por las que los grupos marginalizados utilizan contra-narrativas para construir una realidad más amplia:

- Desafían el status quo (de la dominación cultural).

- Muchos de ellos se centran en construir la comunidad (dentro de comunidades marginadas).

- Pueden abrir nuevas ventanas a la realidad (presentando la posibilidad de múltiples realidades o verdades, que comparten el mismo espacio social o filosófico).

- Pueden acelerar e involucrar conciencia (social).

- Pueden ayudarnos a entender cuándo es el momento de redistribuir el poder.

- Gran parte de la realidad es construida socialmente.
Por supuesto, estos heterodoxos métodos propios de la TCR, así como sus autores, han sido atacados en mayor o menor medida principalmente en la positivista área del Derecho donde surgió (para más detalles sobre el tema ver la amplia discusión sobre autores reaccionarios en Bell, 1995, p. 907-908). Sin embargo, tal como el propio Bell defiende las técnicas, utilizando su particular estilo (p. 908-910), el uso de la TCR podría ser comparado al de los cantos espirituales negros en tiempos de la esclavitud estadounidense: aunque los esclavistas blancos los escuchaban y se sentían entretenidos al pensar que eran inofensivas formas de entretenimiento sin seriedad alguna, en realidad se trataban de códigos y formas de comunicación secreta contra la opresión: avisaban sobre peligros, transmitían información sobre planes de fugas, o simplemente levantaban y fortificaban el ánimo.

En el campo de la organización y representación del conocimiento, los usos de la narrativa y primera persona también han sido utilizados para ilustrar las impresiones y efectos negativos en individuos de grupos marginalizados al utilizar sistemas de organización del conocimiento sesgados (por ejemplo, Grahn, 1984; Penelope 1989; Smiraglia, 2006; de la tierra, 2008). Quizás los ejemplos más ilustrativos sobre narrativas y primera persona, utilizados en relación a estos usos, son recogidos por Hope Olson en The power to name (2002, p. 1-3), incluyendo el relato específico en primera persona de Marielena Fina, publicado originalmente en la revista Cataloging and Classification Quarterly, al descubrir en 1972 que el encabezamiento utilizado para acceder a la información escrita por latinos/as era "bibliotecas y el discapacitado social" ("Libraries and the socially handicapped") después cambiado por "servicio bibliotecario para el discapacitado cultural" ("Library service to the culturally handicapped") (Fina, 1993, p. 269).

Estas técnicas y ejemplos sirven para ilustrar la primera parte de los objetivos de la TCR, la cual es exponer y analizar los casos de racismo sistémico en las instituciones. Para la segunda parte, la erradicación del racismo de estas instituciones y sustitución por alternativas antirracistas, esbozaremos algunos ejemplos de aplicación de la TCR en la CDD y CDU de Brasil.

\section{Aplicación de la TCR a la organización y representación del conocimiento}

Jonathan Furner, en su aplicación de la TCR a la CDD en inglés, indicaba algunas recomendaciones antirracistas para su aplicación a los esquemas de clasificación bibliográficos (Furner, 2007, p. 164, traducción propia). En la pre- 
sente sección se discutirán estos principios para su aplicación a la CDD y CDU de Brasil, así como para ilustrar su extrapolación a otros sistemas de organización del conocimiento:

- Admisión por parte de los diseñadores de que el sesgo en los esquemas de clasificación existe, y de hecho es un inevitable resultado de los modos en los que están actualmente estructurados.

- Reconocimiento de que la adherencia a una política de neutralidad contribuirá poco a la erradicación del sesgo, y de hecho solo puede extender su vida.

- Construcción, recolección y análisis de las expresiones narrativas de los sentimientos, pensamientos y creencias de los usuarios de los sistemas de clasificación que se identifican con poblaciones particulares definidas racialmente.

- El desarrollo de esquemas de clasificación hechos a medida de las necesidades especiales de información de las múltiples y diversas comunidades, permitiendo a los usuarios buscar información sobre las vidas, intereses y actividades de la gente como ellos o con quien ellos se auto-identifican, usando términos de búsqueda con los que son familiares y se sienten confortables.

- La implementación de programas diseñados para cambiar las actitudes de aquellos usuarios potenciales que tienen expectativas muy bajas en los esquemas de clasificación porque esos esquemas son percibidos como inútiles en el mejor de los casos (y fuera de lugar u ofensivos en el peor de los casos) dados sus intereses especiales.

- La adopción de procedimientos y criterios estandarizados para evaluar la utilidad de los esquemas de clasificación para los miembros de las comunidades locales.

Respecto al primer punto, no queda muy claro que los diseñadores de esos sistemas -OCLC en el caso de la CDD en los Estados Unidos, y el UDC Consortium en el caso de la CDU en La Haya- reconozcan la poca idoneidad de estos sistemas para grupos o contextos diversos como es el afrobrasileño. De hecho, la mera denominación de "universal" de la CDU, o las estrategias globales de marketing de la CDD (ver por ejemplo Martínez-Ávila et al., 2012a; 2012b) parecen indicar más bien todo lo contrario. Por otra parte, no deja de ser curioso que muchas de las reivindicaciones hechas por Furner (2007) fueran elaboradas a raíz de su experiencia como editor asistente de la CDD en OCLC, aunque aclarando que "los puntos de vista expresados en el trabajo son propios y no necesariamente representan los de los editores de la CDD o los de OCLC Online Computer Library Center, Inc." (p. 144, traducción propia).

Para paliar esto, un gran avance sería el reconocimiento abierto por parte de los diseñadores de los sistemas (así como su conocimiento y valoración por parte de las instituciones locales y nacionales encargadas de su adopción) de los peligros y problemas que puede suponer utilizar su sistema en países como Brasil para su comunidad afrodescendiente.

El reconocimiento de la no universalidad y no neutralidad de estos esquemas evitaría además la adherencia continuada a fallidos mecanismos de corrección y posturas positivistas que justifican la naturalidad del sesgo mayoritario y rechazan los puntos de vista de los grupos desfavorecidos. Sin embargo, estos grupos ven sistemáticamente silenciada su voz por culpa de unos sistemas de comunicación asimétricos en los que la influencia y exposición de los sistemas sobre los grupos no es proporcional a la capacidad de respuesta y participación de estos usuarios en sus mecanismos de revisión. La reforma o sustitución de estos mecanismos y políticas de participación tanto nacionales como internacionales propiciaría la participación de las comunidades afrodescendientes de Brasil (como el movimiento negro brasileiro y los grupos de investigación sobre temática africana), así como la identificación de estructuras, términos específicos, distorsiones, preconceptos, omisiones, etc., en las estructuras.

Implementando programas de participación más abiertos e inclusivos y sensibles a estos aspectos sociales, tanto en los procesos de revisión de la CDD en OCLC como en los Seminarios Internacionales de la CDU organizados por el UDC Consortium, se permeabilizarian las barreras idiomáticas, económicas y culturales que muchas veces impiden toda posibilidad de participación de estos colectivos.

Finalmente, las autoridades pertinentes en la implantación de los sistemas en Brasil deberán prever y disponibilizar mecanismos de evaluación locales para determinar los efectos de los sistemas sobre estas comunidades y facilitar la participación y amplificación de sus voces en los mecanismos de revisión de las instituciones.

\section{Conclusiones y líneas de actuación futura}

Derrick Bell afirma que "los estándares e instituciones creadas por y que fortalecen el poder blanco deben ser resistidas" (Bell, 1995, p. 901, traducción propia). Una vez identificados esas instancias de racismo en los estándares e instituciones bibliotecarias, el siguiente paso es resistirlas. En el presente trabajo hemos presentado algunas de las líneas teóricas y metodológicas de la TCR aplicadas a las clasificaciones bibliográficas en Brasil. En futuros trabajos de 
investigación se espera presentar y proponer las alternativas específicas para eliminar el racismo y el sesgo en instancias específicas como la religión.

\section{Notas}

(1) Del inglés WEBCHAM: white, ethnically European, bourgeois, Christian, heterosexual, able-bodied, male.

\section{Referencias}

Afolabi, Michael (1992). Spiritual Matters: Provision for Independent African Churches in General Classification Schemes. // International Classification. 19:4, 210-213.

Bell, Derrick A. (1992). Faces at the bottom of the well: the permanence of racism. New York: BasicBooks, 1992.

Bell, Derrick A. (1995). Who's afraid of Citical Race Theory? // University of Illinois Law Review, 893-910.

Berman, Sanford (1971). Prejudices and antipathies: a tract of Library of Congress Subjects Headings concerning people. Metuchen, N. J.: Scarecrow Press, 1971.

Bowker, Geoffrey C.; Star, Susan Leigh. (1999). Sorting things out: classification and its consequences. Cambridge, MA: MIT Press, 1999.

Brock, Andre. (2009). Life on the wire. Information. // Communication \& Society. 12:3 (Jun), 344-363.

Brown, E.M. (1995). The tower of Babel: bridging the divide between critical race theory and "mainstream" civil rights scholarship. // Yale Law Journal 105:2, 513-547.

De la tierra, Tatiana. (2008). Latina Lesbian Subject Headings: the Power of Naming. // Roberto, K. R., (ed.), Radical Cataloging: Essays at the Front. Jefferson, NC: McFarland \& Company, Inc., 2008. 94-102.

Delgado, Richard. (1989). The storytelling for oppositionist and others - a plea for narrative. // Michigan Law Review 87:8, 2411-2441.

Delgado, Richard; Stefancic, Jean. (2001). Critical Race Theory: An Introduction. New York: NYU Press, 2001.

Dunbar, Anthony W. (2006). Introducing critical race theory to archival discourse: getting the conversation started. // Archival Science. 6:1 (Mar) 2006, 109-129.

Fina, Marielena. (1993). The Role of Subject Headings in Access to Information: the Experience of One SpeakingPatron. // Cataloging \& Classification Quarterly 17:1/2, 267-274.

Foskett, Anthony Charles (1971). Misogynists All: A Study in Critical Classification. // Library Resources \& Technical Services. 15:2 (Spring), 117-121.

Foskett, Anthony Charles (1984). Better Dead Than Read: Further Studies in Critical Classification. // Library Resources \& Technical Services. 28 (November/December), 347-359.

Furner, Jonathan. (2007). Dewey Deracialized: A Critical Race-Theoretic Perspective. // Knowledge Organization. 34:3, 144-168.

Furner, Jonathan; Dunbar, Anthony W. (2004). The treatment of topics relating to people of mixed race in bibliographic classification schemes: A critical race-theoretic approach // Mcllwaine, I. C., (ed.). Knowledge organization and the global information society: Proceedings of the Eighth International ISKO Conference. Würzburg, Germany: Ergon Verlag, 2004. 115-120.

García Gutiérrez, Antonio. (2002). Knowledge organization from a "culture of the border": towards a transcultural ethics of mediation. // Lopez-Huertas, María José, (ed.). Challenges in knowledge representations and organiza- tion for the 21st century: integration of knowledge across boundaries: proceedings of the seventh international ISKO conference. Wurzburg: Ergon Verlag, 2002. 516522.

García Gutiérrez, Antonio. (2007). Desclasificados: pluralismo lógico y violencia de la clasificación. Rubí, Barcelona: Anthropos, 2007.

García Gutiérrez, Antonio. (2011). Epistemología de la documentación. Barcelona: Stonberg, 2011.

García Gutiérrez, Antonio; Martínez-Ávila, Daniel. (2014). Critical Organization of Knowledge in Mass Media Information Systems. // Knowledge Organization. 41:3, 205216.

Grahn, Judy. (1984). Another Mother Tongue: Gay Words, Gay Worlds. Boston: Beacon Press, 1984.

Harris, Angela P. (1994). Foreword: The Jurisprudence of Reconstruction // California Law Review. 82, 741-743.

Hjørland, Birger (2007). Arguments for 'The Bibliographical Paradigm'. Some Thoughts Inspired by the New English Edition of the UDC. // Information Research 12:4 paper colis06. http://InformationR.net/ir/12-4/colis06.html (2015-03-05).

Ingram, David (2004). Rights, Democracy, and Fulfillment in the Era of Identity Politics: Principled Compromises in a Compromised World. Lanham, MD: Rowman \& Littlefield, 2004.

Kumasi, Kafi. (2012). Roses in the concrete: a critical race perspective on urban youth and school libraries. /I Knowledge Quest, 40:4 (May/Jun), 32-37.

Martínez-Ávila, Daniel; Olson, Hope A.; Kipp, Margaret. (2012a). New Roles and Global Agents in Information Organization in Spanish Libraries. // Knowledge Organization. 39:2, 125-36.

Martínez-Ávila, Daniel; Kipp, Margaret; Olson, Hope A. (2012b). DDC or BISAC: The Changing Balance Between Corporations and Public Institutions. // Knowledge Organization. 39:5, 309-19.

Martínez Ávila, Daniel; Guimarães, José Augusto Chaves. (2013). Library classifications criticisms: universality, poststructuralism and ethics. // Scire. 19:2 (jul.-dic. 2013), 21-26.

Milani, Suellen Oliveira; Guimarães, José Augusto Chaves; Olson, Hope A. Bias in subject representation: convergences and divergences in the international literature. // Babik, Wieslaw (ed.). Knowledge organization in the 21st century: between historical patterns and future perspectives. Würzburg: Ergon Verlag, 2014. 335-344.

Olson, Hope A. (2000). Difference, Culture and Change: The Untapped Potential of LCSH. // Cataloging \& Classification Quarterly. 29:1/2, 53-71.

Olson, Hope A. (2001a). Sameness and Difference: a Cultural Foundation of Classification. // Library Resources \& Technical Services. 45:3, 115-122.

Olson, Hope A. (2001b). Patriarchal Structures of Subjec Access and Subversive Techniques for Change. // The Canadian Journal of Information and Library Science. 26:2/3, 1-29.

Olson, Hope A. (2002). The Power to Name: Locating the Limits of Subject Representation in Libraries. Dordrecht, Netherlands: Kluwer Academic, 2002.

Olson, Hope A.; Schlegl, Rose (2001). Standardization, Objectivity, and User Focus: A Meta-Analysis of Subject Access Critiques. // Cataloging \& Classification Quarterly. 32:2, 61-80.

Olson, Hope A.; Ward, Dennis B. (1997). Ghettoes and Diaspora in Classification: Communicating Across the Limits. // Frohmann, Bernd (ed.). Communication and Information in Context: Society, Technology, and the Pro- 
fessions. Proceedings of the 25th Annual Conference/ Association canadienne des sciences de l'information: Travaux du 25e congrès annuel. Toronto, Ontario: Canadian Association for Information Science, 1997. 19-31.

Penelope, Julia. (1989). My Life as a Lesbian. // Penelope, Julia; Wolfe, Susan J. (eds.). The Original Coming Out Stories. Freedom, CA: Crossing Press. 43-54.
Smiraglia, Richard P. (2006). Curating and Virtual Shelves: an Editorial. // Knowledge Organization 33:4, 185-187.

Enviado: 2015-03-24. Segunda versión: 2015-05-22. Aceptado: 2015-06-09. 
